and the desired result would follow if any one of these were less than or equal to $\operatorname{Per}(X)$. Actually one can show, for $n=3$, that

$$
3 \operatorname{Per}(X) \geqq \operatorname{Per}\left(X_{1}\right)+\operatorname{Per}\left(X_{2}\right)+\operatorname{Per}\left(X_{3}\right),
$$

but the method offers no hope of generalization.

Even the analogue of the theorem for two arbitrary rows has interesting applications to infinite products and series which seem to be true in the cases tried, but no proof is in sight.

\title{
REFERENCES
}

1. K. Knopp, Theory and applications of infinite series, 2nd English ed., Hafner, New York, 1947.

2. H. J. Ryser, Combinatorial mathematics, Carus Math. Monograph \#14, Wiley, New York, 1963.

Los Alamos Scientific Laboratory, University of California

\section{PERFECT CLOSURES OF RINGS AND SCHEMES'}

\section{MARVIN J. GREENBERG}

0 . In [3], Serre has defined the notion of a perfect variety over a field of characteristic $p>0$. Of course, a perfect variety is, in general, not a variety. The appropriate setting is that of schemes [2]. We show how to construct the perfect closure of a scheme, in particular, of a ring $A$, of characteristic $p$. This amounts to showing that the functor $B \rightarrow \operatorname{Hom}(A, B)$ is representable in the category of perfect rings. We do this by the technique of inductive limits.

1. Let $A$ be a ring (meaning commutative associative unitary ring) of characteristic $p>0, p$ a prime; $p$ is thus the smallest positive integer $n$ such that $n a=0$ for all $a \in A$. Then $A$ has a canonical ring endomorphism, denoted $F$, given by

$$
F(a)=a^{p}, \quad a \in A .
$$

Clearly, $F$ commutes with all homomorphisms of rings of characteristic $p$.

We say $A$ is perfect if $F$ is an automorphism of $A$ (so that every element of $A$ has a unique $p$ th root in $A$ ). For example, finite fields

Presented to the Society, January 23, 1964, under the title Perfect closures of a scheme; received by the editors October 22, 1963.

1 Supported by AFOSR-121-63. 
and algebraically closed fields are perfect.

We say $A$ is $p$-reduced if $F$ has trivial kernel, i.e., $a^{p}=0 \Rightarrow a=0$.

In the general case, the elements of $A$ which are nilpotent of exponent $a$ power of $p$ form an ideal which may be called the $p$-radical of $A$, denoted $A_{p^{\infty}}$. Then $A / A_{p^{\infty}}$ is $p$-reduced.

We wish to solve the following universal problem: Find a pair $\left(A^{*}, \phi\right)$ consisting of a perfect ring $A^{*}$ and a homomorphism $\phi: A \rightarrow A^{*}$ such that, for any other such pair $(B, \psi)$, there is a unique homomorphism $\psi^{*}: A^{*} \rightarrow B$ satisf ying $\psi^{*} \phi=\psi$. As is usual in universal problems, the solution is determined up to unique isomorphism. We call such a pair $\left(A^{*}, \phi\right)$ a perfect closure of $A$. If $A$ is a domain, the existence of a perfect closure is well known and elementary: Simply adjoin to $A$ all $p^{\text {th }}$ roots of elements of $A$ for all $\nu \geqq 1$ (say within some algebraic closure of the quotient field of $A$ ).

2. Every ring $A$ of characteristic $p>0$ has a perfect closure $\left(A^{*}, \phi\right)$. If $x \in A^{*}$, then $x^{p^{\nu}} \in \phi(A)$ for some $\nu \geqq 0$. The homomorphism $\phi$ is injective if and only if $A$ is p-reduced (in fact, the kernel of $\phi$ is $A_{p^{\infty}}$ ).

Proof. Consider the inductive system of rings and homomorphisms $\left(A_{i}, f_{i}\right)_{0 \leq i<\infty}$ given by $A_{i}=A$ and $f_{i}=F$ (where $f_{i}: A_{i} \rightarrow A_{i+1}$ ) for all $i \geqq 0$. Let $A^{*}$ be the inductive limit of this system $[1, \mathrm{p} .9], \phi_{i}: A_{i} \rightarrow A^{*}$ the canonical homomorphism, $\phi=\phi_{0}$.

Let $B$ be any perfect ring, $\psi: A \rightarrow B$ a homomorphism. Define $\psi_{i}: A_{i} \rightarrow B$ by

$$
\psi_{i}(a)=\psi(a)^{1 / p^{i}}, \quad a \in A,
$$

which makes sense because $B$ is perfect. Then $\psi_{0}=\psi$, and $\psi_{i+1} f_{i}=\psi_{i}$ for all $i \geqq 0$. Hence there is a unique homomorphism $\psi^{*}: A^{*} \rightarrow B$ such that $\psi^{*} \phi_{i}=\psi_{i}$ for all $i \geqq 0$, by definition of inductive limit.

Let $g_{i}=f_{i-1} f_{i-2} \cdots f_{0}=F^{i}: A \rightarrow A_{i}$ for any $i \geqq 1$. For any $x \in A^{*}$, there is an $i \geqq 0$ and $y \in A_{i}$ such that $x=\phi_{i}(y)$. Then $y^{p^{i}}=g_{i}(y)$, whence $x^{p^{i}}=\phi_{i} g_{i}(y)=\phi(y)$.

Clearly the kernel of $\phi$ is $A_{p^{\infty}}$, since $\phi(y)=0 \Leftrightarrow g_{i}(y)=0$ for some $i \geqq 1$.

Define the homomorphism $h_{i}: A_{i} \rightarrow A_{i+1}$ for any $i \geqq 0$ to be the identity map of $A=A_{i}=A_{i+1}$. Then $\phi_{i+2} h_{i+1} f_{i}=\phi_{i+1} h_{i}$ for all $i \geqq 0$, whence there is a unique endomorphism $h$ of $A^{*}$ such that $h \phi_{i}$ $=\phi_{i+1} h_{i}$ for all $i \geqq 0$. We claim $h$ is the inverse of $F$ on $A^{*}$ : Given $x \in A^{*}$, choose $i \geqq 1$ and $y \in A_{i}$ such that $x=\phi_{i}(y)$. Then $F(x)=\phi_{i}\left(y^{p}\right)$ $=\phi_{i-1} h_{i}^{-1}(y)$, whence $h F(x)=x$; moreover, $F h(x)=F \phi_{i+1} h_{i}(y)$ $=\phi_{i+1}\left(h_{i}(y)^{p}\right)=\phi_{i+1} f_{i}(y)=\phi_{i}(y)=x$. Thus $A^{*}$ is perfect.

It is convenient to choose once and for all a perfect closure of $A$ 
(say the one we have just constructed), denote it by $A^{1 / p^{\infty}}$ ( $\phi$ understood to be as above), and call it the perfect closure of $A$.

3. We can dualize the above problem by reversing all arrows, i.e., by considering homomorphisms of perfect rings into $A$. A solution $\left(A_{*}, \theta\right)$ to this dual problem may be called a perfect core of $A$. It can be constructed by using projective limits instead of inductive limits (i.e., all $A_{i}=A$ again, but now $F=f_{i}: A_{i+1} \rightarrow A_{i}$ ). The image of $\theta$ is then seen to be $A^{p^{\infty}}$, the subring of those elements having a $p^{\nu}$ th root in $A$ for all $\nu \geqq 1$.

4. It is clear from its definition that the operation of taking the perfect closure is a functor from the category of rings of characteristic $p$ to the category of perfect rings of characteristic $p$. Let $f: A \rightarrow B$ be a homomorphism, $f^{*}: A^{1 / p^{\infty}} \rightarrow B^{1 / p^{\infty}}$ the induced homomorphism, $\phi_{A}, \phi_{B}$ the canonical homomorphisms of $A, B$ (resp.) into $A^{1 / p^{\infty}}$, $B^{1 / p^{\infty}}$ (resp.). We see at once that $f^{*}(x)=0 \Leftrightarrow$ there is a $\nu \geqq 0$ such that $x^{p^{p}} \in \phi_{A}\left(f^{-1}\left(B_{p^{\infty}}\right)\right)$. Hence $f$ injective $\Rightarrow f^{*}$ injective (since $\left.f^{-1}\left(B_{p^{\infty}}\right)=A_{p^{\infty}}\right)$. Moreover, $f^{*}\left(A^{1 / p^{\infty}}\right)$ is the set of all $p^{\nu}$ th roots for all $\nu \geqq 0$ of elements of $\phi_{B}(f(A))$. Hence $f$ surjective $\Rightarrow f^{*}$ surjective.

5. We will need to consider mappings $f: A \rightarrow B$ which are not necessarily ring homomorphisms but are merely additive group homomorphisms respecting $p$ th powers, i.e., additive homomorphisms which commute with $F$. Let us call them additive p-homomorphisms. For example, if $g, h$ are ring homomorphisms $A \rightarrow B, f=g-h$ is no longer a ring homomorphism, but is an additive $p$-homomorphism. In particular, every ring of characteristic $p$ has a canonical additive $p$ endomorphism $\&$ given by

$$
\varphi(x)=x^{p}-x
$$

The kernel of $\varnothing$ is the group of fixed points of $F$.

We then have a more general universal property: If $\psi: A \rightarrow A^{\prime}$ is an additive $p$-homomorphism with values in a perfect ring $A^{\prime}$, then there is a unique additive $p$-homomorphism $\psi^{*}: A^{1 / p^{\infty}} \rightarrow A^{\prime}$ such that $\psi^{*} \phi=\psi$. For $\psi$ induces an additive $p$-homomorphism $\psi^{\prime}: \phi(A) \rightarrow A^{\prime}$, and given $x \in A^{1 / p^{\infty}}, x^{p^{\nu}} \in \phi(A)$, setting

$$
\psi^{*}(x)=\psi^{\prime}\left(x^{p^{\nu}}\right)^{1 / p^{\nu}}
$$

gives the asserted result.

It is then immediate that everything said in $\$ 4$ about a ring homomorphism $f: A \rightarrow B$ remains valid if we only assume $f$ is an additive p-homomorphism. 
In particular, suppose

$$
A \stackrel{f}{\rightarrow} B \stackrel{g}{\rightarrow} C
$$

is an exact sequence of additive $p$-homomorphisms.

Then

$$
A^{1 / p^{\infty}} \stackrel{f^{*}}{\rightarrow} B^{1 / p^{\infty}} \stackrel{g^{*}}{\rightarrow} C^{1 / p^{\infty}}
$$

is again exact: For if $g^{*}(z)=0$, there is a $\nu \geqq 0$ such that $z^{p^{\nu}}$ $\in \phi_{B}\left(g^{-1}\left(C_{p^{\infty}}\right)\right)$, i.e.,

$$
\begin{aligned}
z^{p^{\nu}} & =\phi_{B}(w), \quad w \in B, \\
g(w)^{p^{\mu}} & =0,
\end{aligned}
$$

whence $w^{p \mu}=f(x)$ for some $x \in A$, so that

$$
f^{*} \phi_{A}(x)=\phi_{B}\left(w^{p^{\mu}}\right)=z^{p^{\nu+\mu}}, \quad f^{*} \phi_{A}(x)^{1 / p^{\nu+\mu}}=z .
$$

6. Since perfect closure is a functor, we can then globalize, i.e., extend this functor to the category of preschemes $X$ over $\boldsymbol{Z} / p$ (the finite field with $p$ elements; cf. [2] for facts about preschemes). To any open $U \subset X$, we assign the perfect ring $\Gamma(U)^{1 / p^{\infty}}$, where $\Gamma(U)$ is the ring of sections over $U$ of the structure sheaf $\theta$ of $X$. We then obtain a presheaf on $X$, but by $\$ 5$ this presheaf is a sheaf, i.e., if $\left(U_{i}\right)$ is an open covering of $U$, the sequence

$$
0 \rightarrow \Gamma(U)^{1 / p^{\infty}} \rightarrow \prod_{i} \Gamma\left(U_{i}\right)^{1 / p^{\infty}} \rightarrow \prod_{i, j} \Gamma\left(U_{i} \cap U_{j}\right)^{1 / p^{\infty}}
$$

is exact.

We denote this sheaf by $0^{1 / p^{\infty}}$, and call the ringed space $\left(X, \theta^{1 / p^{\infty}}\right)$ the perfect closure of $X$, briefly $X^{1 / p^{\infty}}$. We have a canonical morphism of ringed spaces $\Phi: X^{1 / p^{\infty}} \rightarrow X$, which is the identity on the underlying topological space.

7. Let $S$ be a multiplicative submonoid of $A$, i.e., $1 \in S$, and $S$ is stable under multiplication. Let $S^{1 / p^{\infty}}$ be the perfect closure of $S$ in $A^{1 / p^{\infty}}$, i.e., the set of all $x \in A^{1 / p^{\infty}}$ such that $x^{p^{p}} \in \phi(S)$ for some $\nu \geqq 0$. Then the rings $\left(A_{S}\right)^{1 / p^{\infty}}$ and $\left(A^{1 / p^{\infty}}\right)_{S^{1 / p^{\infty}}}$ are canonically isomorphic (cf. [2] for rings of fractions): There is an obvious homomorphism

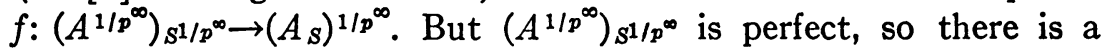
canonical homomorphism in the other direction which is inverse to $f$.

Perfect closure commutes with filtering inductive limits: This follows immediately from the fact that a filtering inductive limit of perfect rings is perfect. Thus the stalk of $X^{1 / p^{\infty}}$ at a point $x$ is $\left(\theta_{x}\right)^{1 / p^{\infty}}$. If we 
take an affine neighborhood $\operatorname{Spec} A$ of $x$, so that $x$ is a prime ideal in $A$, and $S=A-x$, then $\left(\Theta_{x}\right)^{1 / p^{\infty}}=\left(A^{1 / p^{\infty}}\right)_{S^{1 / p^{\infty}}}$.

To each ideal $\mathfrak{a}$ in $A$, associate the ideal $\mathfrak{a}^{1 / p^{\infty}}$ in $A^{1 / p^{\infty}}$ defined in the obvious way. We obtain an epimorphism of the lattice of ideals in $A$ onto the lattice of perfect ideals in $A^{1 / p^{\infty}}$ (ideals stable under extraction of $p$ th roots). If we define the $p$-radical of an ideal $a$ to be the ideal ${ }^{p} \sqrt{ } \mathfrak{a}$ of all elements in $A$ having some $p$ th power in $\mathfrak{a}$, then $\mathfrak{a}^{1 / p^{\infty}}=\mathfrak{b}^{1 / p^{\infty}} \Leftrightarrow p \sqrt{ } \mathfrak{a}=p \sqrt{ } \mathfrak{b}$. In particular, the lattice of prime ideals in $A$ is isomorphic to the lattice of prime ideals in $A^{1 / p^{\infty}}$, so that $\operatorname{Spec} A^{1 / p^{\infty}} \rightarrow \operatorname{Spec} A$ is a homeomorphism.

If $\mathfrak{p}$ is a prime ideal in $A, S=A-\mathfrak{p}$, one checks at once that $S^{1 / p^{\infty}}$ $=A^{1 / p^{\infty}}-\mathfrak{p}^{1 / p^{\infty}}$. Thus we can identify $\operatorname{Spec} A^{1 / p^{\infty}}$ with $(\operatorname{Spec} A)^{1 / p^{\infty}}$, so that the perfect closure of a prescheme is again a prescheme, having a structure sheaf of perfect local rings. We can call such a prescheme a perfect prescheme. The couple $\left(X^{1 / p^{\infty}}, \Phi\right)$ is then universal with respect to morphisms of perfect preschemes into $X$. The category of perfect preschemesis stable under products, since the tensor product of two perfect rings is perfect, and perfect closure commutes with products. It follows that the perfect closure of a group scheme is again a group scheme.

A perfect prescheme has a canonical family $\left(F^{n}\right)_{n \in Z}$ of automorphisms which are the identity on the base space.

\section{BibliogRAPHY}

1. R. Godement, Theorie des faisceaux, Hermann, Paris, 1958.

2. A. Grothendieck and J. Dieudonné, Elements de gémetrie algêbrique. I, Inst. Hautes Etudes Sci. Publ. Math. No. 4 (1960), 1-228.

3. J. P. Serre, Groupes proalgebriques, Inst. Hautes Etudes Sci. Publ. Math. No. 7 (1960), 1-67.

University of California, Berkeley 